\title{
Lattice thermal properties of graphane: Thermal contraction, roughness, and heat capacity
}

\author{
M. Neek-Amal ${ }^{1}$ and F. M. Peeters ${ }^{2}$ \\ ${ }^{1}$ Department of Physics, Shahid Rajaee Teacher Training University, Lavizan, Tehran 16785-136, Iran \\ ${ }^{2}$ Departement Fysica, Universiteit Antwerpen, Groenenborgerlaan 171, B-2020 Antwerpen, Belgium
}

(Received 10 May 2011; published 30 June 2011)

\begin{abstract}
Using atomistic simulations, we determine the roughness and the thermal properties of a suspended graphane sheet. As compared to graphene, we found that (i) hydrogenated graphene has a larger thermal contraction, (ii) the roughness exponent at room temperature is smaller, i.e., $\simeq 1.0$ versus $\simeq 1.2$ for graphene, (iii) the wavelengths of the induced ripples in graphane cover a wide range corresponding to length scales in the range $30-125 \AA$ at room temperature, and (iv) the heat capacity of graphane is estimated to be $29.32 \pm 0.23 \mathrm{~J} / \mathrm{mol} \mathrm{K}$, which is $14.8 \%$ larger than that for graphene, i.e., $24.98 \pm 0.14 \mathrm{~J} / \mathrm{mol} \mathrm{K}$. Above $1500 \mathrm{~K}$, we found that graphane buckles when its edges are supported in the $x-y$ plane.
\end{abstract}

DOI: 10.1103/PhysRevB.83.235437

PACS number(s): 65.40.De

\section{INTRODUCTION}

Graphane (GA), a two-dimensional covalently bonded hydrocarbon, was first predicted from $a b$ initio calculation by Sluiter $e t a l .{ }^{1}$ and recently rediscovered. ${ }^{2}$ In a recent experiment, Elias et al. ${ }^{3}$ demonstrated the fabrication of GA from a graphene (GE) membrane through hydrogenation, which was found to be reversible. Density functional theory (DFT) and molecular-dynamics simulations employing different force fields were carried out to study the structural and electronic properties of both GA and GE. ${ }^{4,5}$

Hydrogen $(\mathrm{H})$ atoms are chemically bound to the carbon (C) atoms on alternating sides of the membrane (chairlike conformer), which causes a local buckling of the membrane. Such deformations for small membrane sizes $(<1 \mathrm{~nm})$ have been recently reported. ${ }^{6}$ In the early stages of the hydrogenation process, membrane shrinking and extensive membrane corrugations occur due to the formation of a significant percentage of uncorrelated $\mathrm{H}$ frustrated domains. ${ }^{6}$

The morphology of perfect GA, and its comparison with a perfect GE membrane, has not yet been investigated for large samples. Ab initio calculations are restricted to small unit cells, therefore we will use atomistic simulations to show the main differences between the morphology of a large sample of GA and GE. Our atomistic simulations are based on the second generation of the Brenner potential ${ }^{7}$ [reactive empirical bond order (REBO)], which includes the interaction with third nearest neighbors via a bond-order term that is associated with the dihedral angles. Therefore, such a potential is suitable for various atomistic simulation purposes, including the calculations of the lattice thermal properties (phonon dispersion, ${ }^{8}$ elastic moduli, ${ }^{9}$ thermal conductivity, ${ }^{10}$ etc.) of carbon nanotubes, graphene, and hydrocarbons. Nevertheless, there are a few shortcomings in the REBO potential that are important when modeling processes involve energetic atomic collisions (because both Morse-type terms approach finite values at very small atomic distance), and when the neglect of a separate $\pi$ bond contribution leads to the overbinding of radicals. Our study is not affected by these shortcomings. Of course, the electronic properties of GE and GA and the thermodynamic properties at low temperatures (because of, e.g., quantum zero energy) are beyond the REBO's ability. In those situations, ab initio calculations, particularly those based on DFT, are extremely useful. Ab initio molecular-dynamics simulation (e.g., Car-Parrinello molecular dynamics ${ }^{11}$ ) is a highly appreciated theory that eliminates the force-field-based restrictions, but its main disadvantage is that it is only applicable for small-size systems (typically $N<500$ atoms).

Here we study relatively large systems and consider the thermal and structural properties of GE and GA above $50 \mathrm{~K}$, and we show that for a GA sample with size $183 \AA \times 185 \AA$ containing 22400 atoms, the roughness, the induced ripples structure, and the total energies are very different from a GE membrane. It has been shown that the ripples in GE strongly affect its thermoelectronic properties. ${ }^{12}$ Temperature effects are studied and we found that the thermal contraction of a suspended GA is larger than the one for GE. Moreover, the calculated roughness exponent indicates that GA is more rough than GE even at room temperature. From our simulations, we predict that the ripples in GA affect its thermoelectronic properties much more than for GE.

This paper is organized as follows. In Sec. II, we introduce the atomistic model and the simulation method. Section III contains our main results for both graphene and graphane. Results for thermal contraction, roughness, and heat capacity for different temperatures are presented and compared to available experimental results. In Sec. IV, we conclude the paper.

\section{METHOD AND MODEL}

Classical atomistic molecular-dynamics (MD) simulation is employed to simulate large flakes of GE and GA at various temperatures. The second generation of Brenner's bond-order potential is employed, which is able to describe covalent $s p^{3}$ bond breaking and the formation ofssociated changes in atomic hybridization within a classical potential. ${ }^{7}$ The Brenner potential (REBO) terms were taken as $E_{P}=$ $\sum_{i} \sum_{j>i}\left[V^{R}\left(r_{i j}\right)-B_{i j} V^{A}\left(r_{i j}\right)\right]$, where $E_{P}$ is the average binding energy, and $V^{R}$ and $V^{A}$ are the repulsive and attractive term, respectively, where $r_{i j}$ is the distance between atoms $i$ and $j . B_{i j}$ is called the bond order factor, which includes all many-body effects. $B_{i j}$ depends on the local environment of the bond, i.e., the bond and torsional angles, the bond lengths, and the atomic coordination in the vicinity of the bond. This feature allows the Brenner potential to predict correctly the 

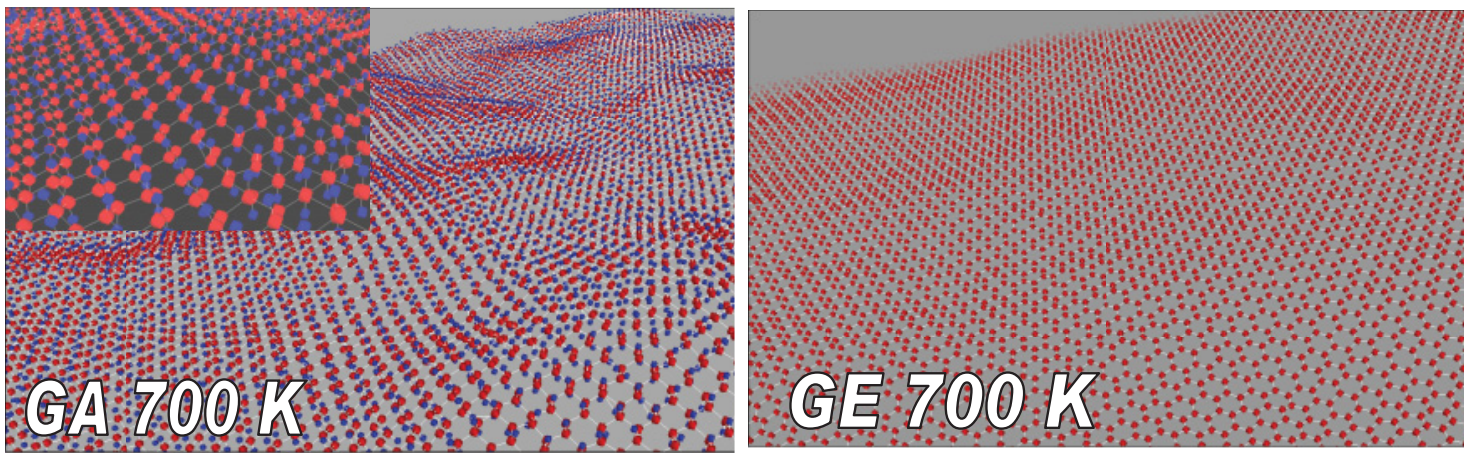

FIG. 1. (Color online) Two snapshots of suspended graphane (GA) and graphene (GE) at $700 \mathrm{~K}$. The inset is a zoomed region that shows a regular alignment of $\mathrm{C}-\mathrm{H}$ bonds.

configurations and energies for many different hydrocarbon structures and the correct hybridization.

Initially the coordinates of all carbon atoms in GA (GE) are put in a flat surface of a honeycomb lattice with nearestneighbor distance equal to $a_{0}=0.153 \mathrm{~nm}(0.142 \mathrm{~nm})$. The hydrogen atoms are alternatively put on top and bottom of the carbons, i.e., $(\mathrm{CH})_{n}$ with $n=11200$ (chairlike model for GA, ${ }^{2}$ see the inset in the left panel of Fig. 1). To make sure that the second generation of the Brenner potential gives the correct force field for our thermomechanical studies of the GA sheet, we performed extra simulations in which we started with a nonoptimum bond length of $a_{0}=0.142 \mathrm{~nm}$. Already after only a few time steps, the correct optimum GA lattice spacing, i.e., $a_{0} \sim 0.153 \mathrm{~nm}$, was found, confirming the ability of the used potential for GA simulations.

The considered systems are square sheets with dimensions $183 \AA \times 185 \AA$ for GA and $170 \AA \times 170 \AA$ for GE in the $x$ and $y$ directions, where we considered both armchair and zigzag edges. The number of atoms in GA (GE) is $n=22400(n / 2)$. We simulated the system at nonzero temperatures (above $50 \mathrm{~K}$ ) by employing a Nosé-Hoover thermostat. In order to mimic the experimental setup, we prevented motion along the $z$ direction at the ends of the system along $x$. This is realized in practice by fixing a row of atoms at both longitudinal sides. This supported boundary condition prevents the drift (of the ends of the sheet) in the $z$ direction. Motion in the $x-y$ plane is allowed, which allows the system to relax and to exhibit lattice contraction and expansion.

Before starting the sampling, we let the system find its true size and temperature during the first 5 ps. During this process, GA and GE change their longitudinal length so that we always observe lattice contraction, implying that GA and GE shrink through surface corrugation (i.e., creating ripples). ${ }^{13}$ Figure 1 shows two snapshots of GA and GE relaxed at $700 \mathrm{~K}$. The C-H bonds (except at the boundaries) are almost parallel, which is a consequence of lateral $\mathrm{H}-\mathrm{H}$ repulsion.

\section{RESULTS AND DISCUSSION}

\section{A. Equilibrium length and thermal contraction}

After equilibrating, we start to record the longitudinal size (in the $x-y$ plane along the $x$ axis) of the system for 2 ps. Averaging over the lengths during the sampling process gives the average length of the system, i.e., $\langle L\rangle$, at the given temperature. Figure 2(a) shows the thermal contraction coefficient, i.e., $\gamma_{L}=\frac{d L}{L_{0} d T}$, versus temperature, where $L_{0}=183 \AA\left(L_{0}=\right.$ $170 \AA$ ) is the initial length of GA (GE) (for a flat sheet at $T=$ $0 \mathrm{~K})$ and $d L=\langle L\rangle-L_{0}$. As we see, $\gamma$ increases with temperature and is always negative in the studied temperature range. Surprisingly, GA has a larger contraction with temperature than GE. These curves are fitted to $\alpha+\beta / T$, where $\alpha=22 \pm$
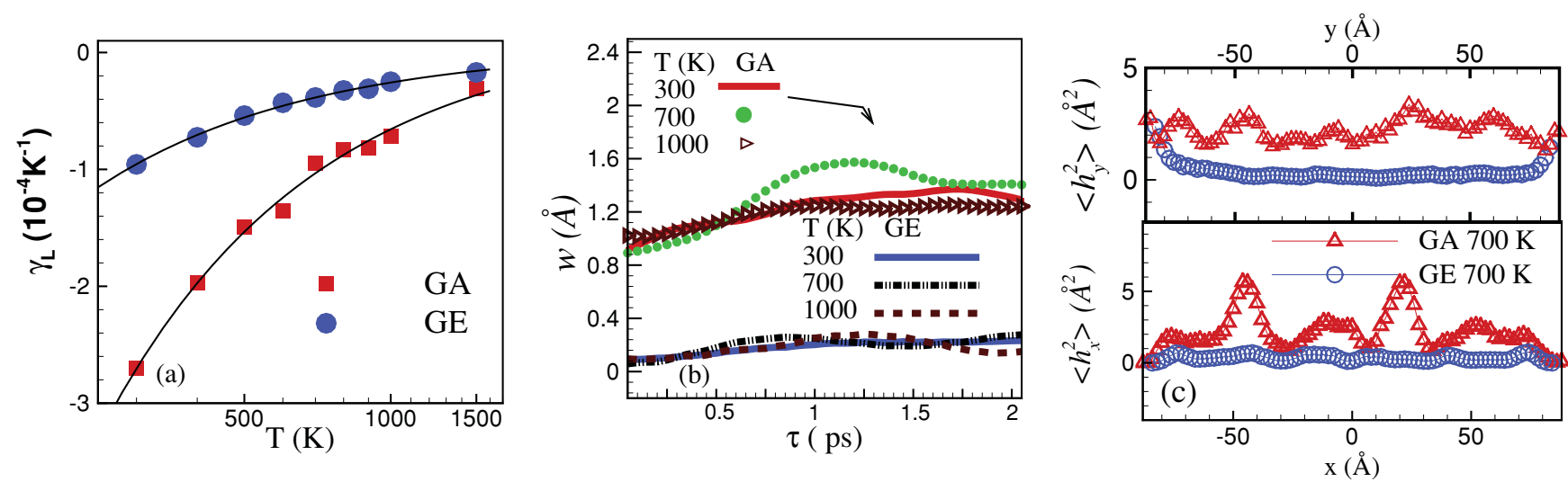

FIG. 2. (Color online) (a) Temperature dependence of the thermal contraction coefficient of a square sheet of GA and GE subjected to longitudinal supported boundary and lateral free-boundary conditions. Solid curves are fits according to $\alpha+\beta / T$. (b) Roughness of GA and GE as a function of time. (c) The variation of $\left\langle h_{x}^{2}\right\rangle$ (averaged over lateral size) and $\left\langle h_{y}^{2}\right\rangle$ (averaged over longitudinal size) at $700 \mathrm{~K}$. 
$5 \times 10^{-6} \mathrm{~K}^{-1}, 4 \pm 1 \times 10^{-6} \mathrm{~K}^{-1}$, and $\beta=-877.10 \pm 29$ and $-298.8 \pm 6$ for GA and GE, respectively [solid curves in Fig. 2(a)].

At present, a few studies exist on the negative thermalexpansion coefficient (TEC) of GE, but none are available for GA. The actual values of the TEC's for GE differ from one study to the other depending on, e.g., the size and the boundary conditions. ${ }^{14}$ Mounet and Marzari ${ }^{15}$ presented a study of the thermodynamic and structural properties of carbon-based structures (graphite, diamond, and GE) using generalizedgradient approximation (GGA) DFT calculations. To calculate the finite-temperature thermal expansion and heat capacity, a quasiharmonic approximation (QHA) was employed. ${ }^{14,15}$ Using the DFT results for the phonon-dispersion relation and minimizing the QHA free energy with respect to the lattice parameter $\left(\left.\frac{\partial F}{\partial a}\right|_{T}=0\right)$, they found the linear thermal expansion of the lattice parameter, $a$, for graphite, GE, and diamond. The linear thermal expansion for the lattice parameter of GE, $\gamma_{a}=\frac{1}{a} \frac{\partial a}{\partial T}$, where $a=\sqrt{3} a_{0}$, was found to be negative and about $\gamma_{a} \simeq-0.35 \times 10^{-5} \mathrm{~K}^{-1}$ at room temperature. QHA + GGA includes only weak anharmonicity, but it includes quantum effects and zero point energy. The strong anharmonic coupling of the bending and stretching modes in GE, which are essential for the GE stability, are accurately described by classical empirical potentials at nonzero temperatures. ${ }^{16}$ In our study, the negative thermal contraction is reported for the length, $L$, of GA and GE samples. Since ripples appear in GE and GA membranes, we expect a contraction of the length of the systems. $\gamma_{L}$ is not directly related to $\gamma_{a}$. In fact, $d L$ is the variation of the measured length along the $x$ direction, while $d a$ is the variation of the lattice parameter not necessarily in the $x-y$ plane. Therefore, our results for the contraction of the GE's length are not directly related to those of QHA + GGA for contraction of the lattice parameter.

In a recent experiment, ${ }^{17}$ the morphology of different graphene membranes (micron size membranes) suspended across trenches on $\mathrm{Si} / \mathrm{SiO}_{2}$ substrates was investigated using atomic force microscopy (AFM) and scanning tunneling microscopy (STM). TEC's were found to be about $\gamma_{\exp } \sim-0.7 \times 10^{-5} \mathrm{~K}^{-1}$ at room temperature. With increasing temperature, the measured TEC's approach zero nonlinearly. Here, we find a similar contraction in the longitudinal size (e.g., $\gamma_{L} \sim-9.0 \times 10^{-5} \mathrm{~K}^{-1}$ at $T=300 \mathrm{~K}$ for GE) which approaches zero with increasing temperature but which is an order of magnitude larger. To compute the thermal contraction, we calculated the longitudinal size (not the arc length, as done in the experiment ${ }^{17}$ ). There are important differences between our approach and those from the above-mentioned experiment. First, we use the initial length (as a reference for the length) of our samples as the length of the sheet at zero temperature. Second, the sample size is different. Third, there is no substrate in our study, which could reduce the contraction. Our suspended sample is free to drift in the $x-y$ plane, while in the experiment the substrate prevents free drift in the $x-y$ plane in addition to movement along the $z$ direction, which causes a lower contraction. Notice that $\gamma_{L}<\gamma_{\exp }<\gamma_{a}$. We found that in thermal equilibrium and under supported boundary conditions, the equilibrium size of GE is longer

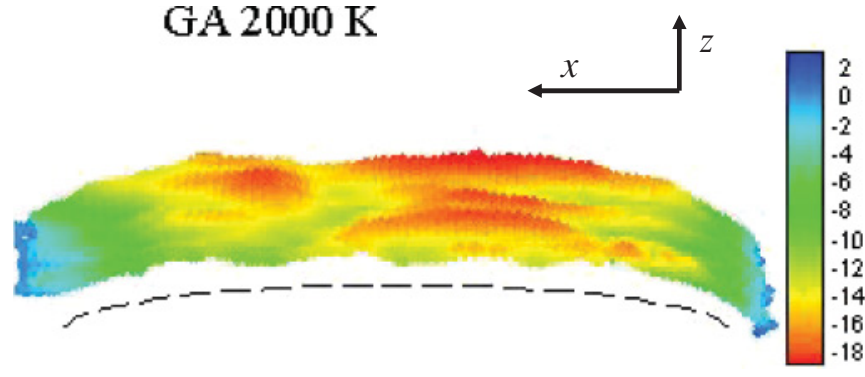

FIG. 3. (Color online) Buckling of graphane at 2000 K. Dashed curve shows the convex shape of graphane.

than GA. Therefore, the GA surface is much more corrugated than GE.

The larger contraction of GA is due to the larger amplitude of the ripples as compared to GE. The reason is that hydrogen atoms that are below and above the sheet attract or repel the carbon atoms and push them in different directions (i.e., random thermal fluctuations of the hydrogens at finite temperature). Therefore, we expect an increasing randomness of the GA sheet and the formation of different patterns of ripples in a GA sheet and a larger corrugation. The thickness of GA is larger than GE, hence one expects for such a thicker material an ordinary positive thermal expansion (or at least smaller thermal contraction with respect to GE). However, we found negative thermal contraction for GA, which is larger than the one for GE. It is interesting to note that above $1500 \mathrm{~K}$ we observe a buckling of GA. Figure 3 shows a snapshot of a buckled GA sheet at $2000 \mathrm{~K}$. A similar buckling was observed in experiments on suspended GE (Ref. 17) and in a circular GE sheet subjected to radial strain. ${ }^{18}$ Therefore, increasing temperature induces larger axial strains in GA.

\section{B. Roughness}

Figure 2(b) shows the average square root out-of-plane deviation of the carbon atoms (i.e., static roughness, i.e., $w=\sqrt{\left\langle h^{2}\right\rangle-\langle h\rangle^{2}}$ ) versus time for three typical temperatures. For long times, $w$ fluctuates around $0.2 \AA$ for GE and $1.3 \AA$ for GA, which is 6.5 times larger. Fig1ure 2(c) shows the variation of $\left\langle h_{x}^{2}\right\rangle$ (where the average is over the lateral size of the system) and $\left\langle h_{y}^{2}\right\rangle$ (where the average is over the longitudinal size of the system) for GE and GA at $T=700 \mathrm{~K}$. Because of the specific boundary condition, the ripples appear in the $x$ direction, while in the $y$ direction the fluctuations are much smaller and for GE they are almost zero. This leads to a larger randomness in GA, which relates to a less stiff material as compared to GE. ${ }^{5}$ This is illustrated in Fig. 4, which shows the corresponding contour plot for GA and GE at $T=300$ and $700 \mathrm{~K}$. Notice the difference in scale for the amplitude. For example, for $\mathrm{GA}$ at $700 \mathrm{~K}$, the amplitude of the ripples is in the $[-4,4] \AA$ A range and $[-2,2] \AA$ A for GE. In the lateral boundaries, we see larger amplitude variations. The free boundaries in GE exhibit larger free vibrations than GA. This can also be inferred from the top panel of Fig. 2(c). Notice that the typical wavelengths $(\lambda)$ for the ripples in GA are very different as compared to that of GE. Figure 5 shows the amplitude of the Fourier transform of $h_{x},\left\langle\left|h_{q}\right|^{2}\right\rangle$ (with an ensemble average taken over 60 samples), for GA and GE as a function of the 

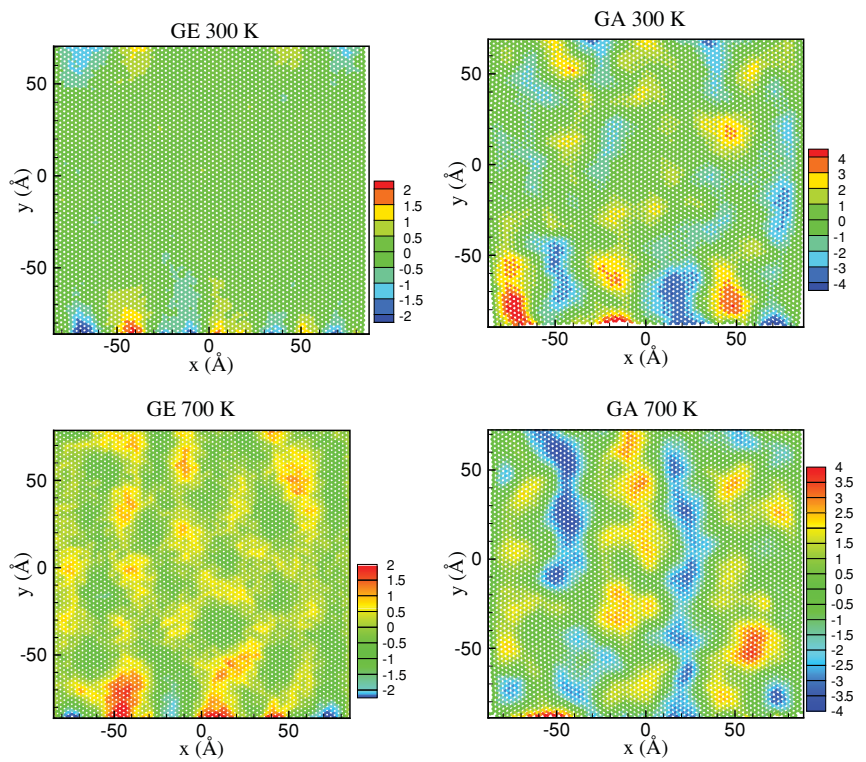

FIG. 4. (Color online) Contour plot of the $z$ position of the carbon atoms of GA and GE at 300 and $700 \mathrm{~K}$ corresponding to the situation of Fig. 2(c). The lateral edges are free while the longitudinal edges are not allowed to move in the $z$ direction.

wavelength along the $x$ direction (taken in the middle of the system around $y=0), q_{x}=\frac{2 \pi}{\lambda}$ for two different temperatures. Independent of temperature, the ripples in GA span a wider range of wavelengths. For example, they span length scales in the 30-125 and 25-140 $\AA$ ranges for $T=300$ and $700 \mathrm{~K}$, respectively. This compares with ripples in GE having a typical length scale around $60 \AA$ for $T=700 \mathrm{~K}$, while for $T=300 \mathrm{~K}$ the membrane is almost flat.

Next we estimate the roughness exponent $\chi$ of GA (where only the carbon atoms are considered), which is obtained from the second-order structure function, i.e., $S(\delta)=\mid\langle h(x+\delta)-$ $h(x)\rangle\left.\right|^{2}$, which scales as $\delta^{x}$ (Ref. 19), where $\delta$ counts the atoms along the $x$ direction, e.g., $\delta=4$ refers to the fourth atom in the armchair direction ( $\delta$ is typically $2 \AA$ ). Figure 6(a) shows the variation of $S(\delta)$ versus $\delta$ for GA and GE for two temperatures. Notice that $S(\delta)$ for GA is almost an order of magnitude larger than for $\mathrm{GE}$, indicating the larger corrugation.

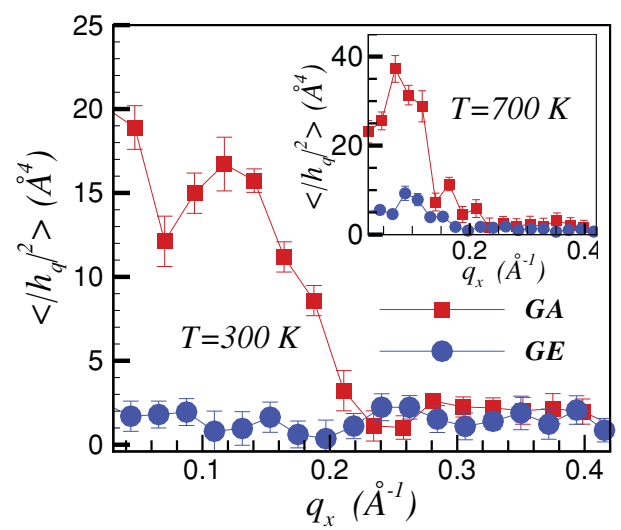

FIG. 5. (Color online) The amplitude of the Fourier transform of $h_{x}$ of GA and GE atoms along the $x$ direction (averaged over 60 samples) for two temperatures $T=300$ and $700 \mathrm{~K}$ (inset).
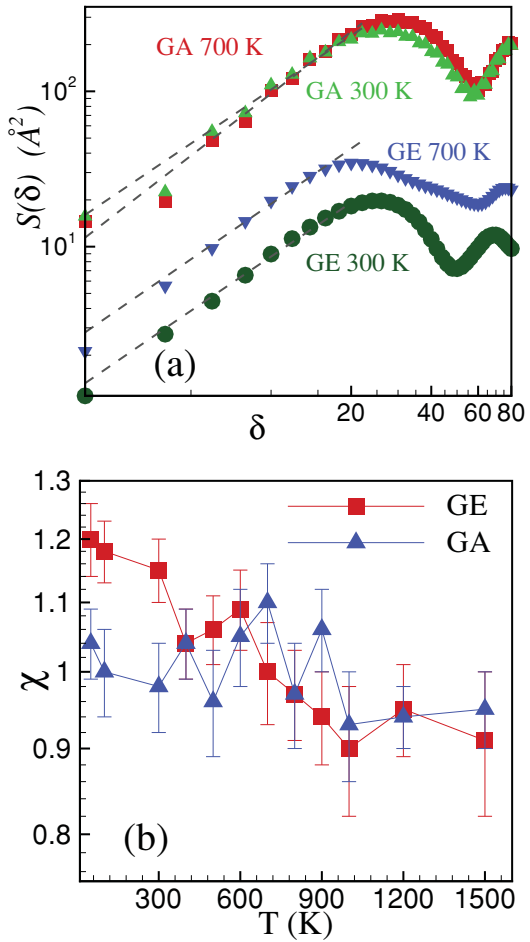

FIG. 6. (Color online) (a) Variation of the structure function vs $\delta$ (log scale) for GE and GA at 300 and $700 \mathrm{~K}$. Here $\delta$ counts the atoms along the armchair direction and is about $2 \AA$. Dashed lines are fits to $\delta^{x}$. (b) Roughness exponent as a function of temperature for GA and GE.

$S(\delta)$ increases with $\delta$ up to some critical $\delta$ values, $\delta_{c}$, which is related to the above-mentioned characteristic length scale of the ripples. For $\delta<\delta_{c}$, the height of the atoms is correlated while it becomes uncorrelated for $\delta>\delta_{c}$. The slope of $S(\delta)$ gives $\chi$, whose temperature dependence is shown in Fig. 6(b). Below room temperature, GE has a larger $\chi(\simeq 1.2)$, which implies that in this temperature range the GE membrane has a smoother surface as compared to GA. However, at high temperatures both systems approach the situation with random height fluctuations, i.e., $\chi \sim 1$. Indeed, one would expect that the presence of the $s p^{3}$ bonds in GA (making GA effectively a much thicker material than GE) decreases the roughness with respect to GE, while we found that it increases.

\section{Heat capacity}

Figure 7(a) shows the variation of the potential energy per atom $\left(E_{P}\right)$ for $\mathrm{C}$ atoms in $\mathrm{GE}, \mathrm{C}$ atoms in $\mathrm{GA}$, and $\mathrm{H}$ atoms in GA versus temperature (all data points were obtained by averaging over an ensemble of 150 samples). The potential energy is a measure of the binding energy (or bond energy), i.e., $B E=-E_{P}$. The binding energy of the $\mathrm{C}$ atoms in GA is larger than that in GE. The right scale in Fig. 7(a) is for the potential energy of $\mathrm{H}$ atoms in GA. The variation of the potential energy of $\mathrm{H}$ atoms with temperature is smaller than that for $\mathrm{C}$ atoms in GA. Note that an accurate study of the GA binding energies at low temperature requires ab initio molecular-dynamics simulations, where quantum fluctuations are included. At low temperature, where the difference between energy of two quantum states of the system is larger than the thermal 

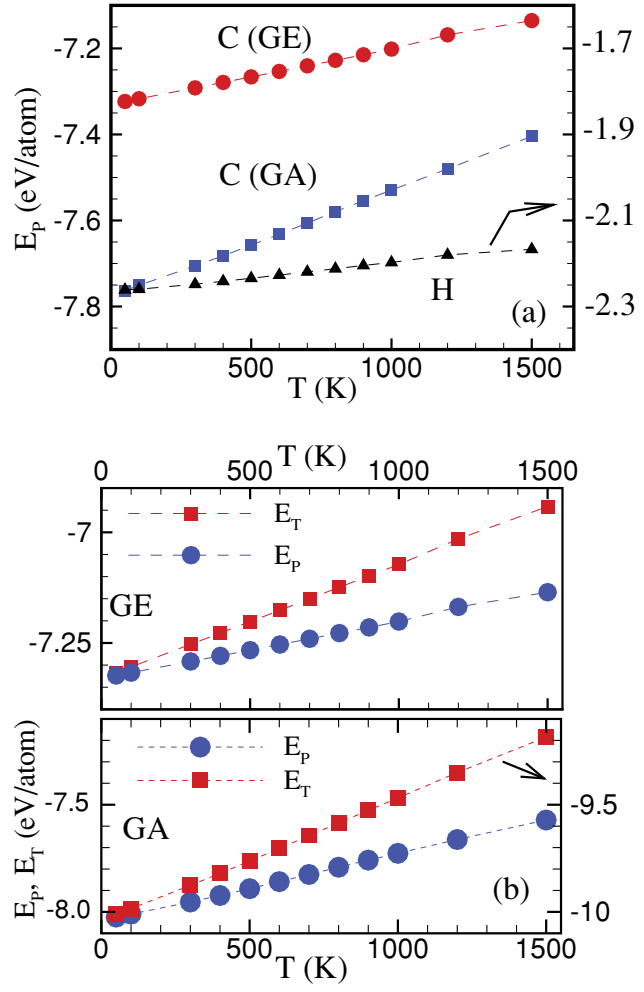

FIG. 7. (Color online) (a) Variation of the potential energy $\left(E_{P}\right)$ per atom vs temperature for both $\mathrm{C}$ atoms in $\mathrm{GA}$ and $\mathrm{GE}$ and $\mathrm{H}$ atoms in GA. The right scale is for $\mathrm{H}$ atoms and the left scale is for $\mathrm{C}$ atoms. (b) Variation of total energy $\left(E_{T}=E_{P}+E_{K}\right)$ per atom vs temperature for both GA and GE. The right scale is for GA. The error bars are less than $0.005 \mathrm{eV} /$ atom.

energy, many-body effects and the chemical covalent bond energies become sensitive to the quantum states. Here we found that the sum of the binding energy of $\mathrm{C}-\mathrm{H}$ bonds at $50 \mathrm{~K}$ is $5.012 \mathrm{eV}$ per atom, which is comparable to the recently reported value at $T=0 \mathrm{~K}$, i.e., $5.19 \mathrm{eV}$ per atom using DFT calculations. ${ }^{20}$ The extrapolation of our data to $T=0 \mathrm{~K}$ gives $5.02 \mathrm{eV}$ per atom, which indicates that quantum corrections are of order $0.17 \mathrm{eV}$ per atom, or about 3.3\%. Extrapolation in Fig. 7(a) for the binding energy of $\mathrm{C}$ atoms in $\mathrm{GE}$ gives $7.33 \mathrm{eV} /$ at (for all reported energies, the error bars are less than $0.005 \mathrm{eV} / \mathrm{at})$.
Figure 7(b) shows the total energy per atom (the sum of potential and kinetic energy of $\mathrm{C}$ and $\mathrm{H}$ atoms $E_{T}=$ $E_{P}+E_{K}$ ) versus temperature [potential energies are also shown in Fig. 7(b) for comparative purposes]. The right scale in Fig. 7(b) is for the total energy of GA. At a temperature around $1500 \mathrm{~K}$, we observe evaporation of $\mathrm{H}$ atoms at the free edges. The total energy varies linearly with temperature and gives the corresponding lattice contribution to the molar heat capacity at constant volume (the average size of the system after relaxation is taken constant), $C_{V}=\frac{d E_{T}}{d T}$, which for GE is $24.98 \pm 0.14 \mathrm{~J} / \mathrm{mol} \mathrm{K}$ and for GA is $29.32 \pm$ $0.23 \mathrm{~J} / \mathrm{mol} \mathrm{K}$. This is comparable to the proposed classical heat capacity at constant volume, i.e., $C_{V}=3 \Re \simeq 24.94$ $\mathrm{J} / \mathrm{mol} \mathrm{K}$, i.e., the Dulong-Petit limit, where $\mathfrak{R}$ is the universal gas constant. The larger heat capacity for GA is due to the extra storage of vibrational energy in the $\mathrm{C}-\mathrm{H}$ bonds. Our result for GE is in agreement with those obtained from Monte Carlo simulations of Ref. 21 but with smaller error bars. The reported heat capacity for GE in Ref. 21 is $C_{V} \simeq$ $25 \pm 1.0 \mathrm{~J} / \mathrm{mol} \mathrm{K}$ for $T=1000 \mathrm{~K}$. The used size for the GE samples in Ref. 21 is $13 \%$ smaller than those in the present study.

\section{CONCLUSION}

In this paper, we studied the thermal properties of suspended graphane and compared the obtained results to those found for graphene. We found that the roughness, amplitude, and wavelengths of the ripples are very different. The thermal contraction effect for graphane is larger than that for graphene. Above $1500 \mathrm{~K}$, we found that graphane is buckled and starts to lose $\mathrm{H}$ atoms at the edges of the membrane. Roughness in graphane is greater than that in graphene, and the roughness exponent in graphene decreases versus temperature (from 1.2 to 1.0) while for graphane it stays around 1.0, implying random uncorrelated roughness. Fourier analysis of the height of the $\mathrm{C}$ atoms showed that the ripples in graphane exhibit a larger range of length scales as compared to those for graphene. The heat capacity of graphane is found to be $14.8 \%$ larger than that for graphene.

\section{ACKNOWLEDGMENTS}

This work was supported by the Flemish Science Foundation (FWO-Vl) and the Belgium Science Policy (IAP).
${ }^{1}$ M. H. F. Sluiter and Y. Kawazoe, Phys. Rev. B 68, 085410 (2003).

${ }^{2}$ J. O. Sofo, A. S. Chaudhari, and G. D. Barber, Phys. Rev. B 75, 153401 (2007).

${ }^{3}$ D. C. Elias, R. R. Nair, T. M. G. Mohiuddin, S. V. Morozov, P. Blake, M. P. Halsall, A. C. Ferrari, D. W. Boukhvalov, M. I. Katsnelson,

A. K. Geim, and K. S. Novoselov, Science 323, 610 (2009).

${ }^{4}$ D. K. Samarakoon and X.-Q. Wang, ACS Nano 3, 4017 (2009).

${ }^{5}$ O. Leenaerts, H. Peelaers, A. D. Hernandez-Nieves, B. Partoens, and F. M. Peeters, Phys. Rev. B 82, 195436 (2010).

${ }^{6}$ M. Z. S. Flores, P. A. S. Autreto, S. B. Legoas, and D. S. Galvao, Nanotechnology 20, 465704 (2009).
${ }^{7}$ D. W. Brenner, O. A. Shenderova, J. A. Harrison, S. J. Stuart, B. Ni, and S. B. Sinnot, J. Phys. Condens. Matter 14, 783 (2002).

${ }^{8}$ L. Lindsay and D. A. Broido, Phys. Rev. B 81, 205441 (2010).

${ }^{9}$ Q. Lu, M. Arroyo, and R. Huang, J. Phys. D 42, 102002 (2009).

${ }^{10}$ A. V. Savin, B. Hu, and Y. S. Kivshar, Phys. Rev. B 80, 195423 (2009).

${ }^{11}$ R. Car and M. Parrinello, Phys. Rev. Lett. 55, 2471 (1985).

${ }^{12}$ F. Guinea, B. Horovitz, and P. Le Doussal, Phys. Rev. B 77, 205421 (2008); I. F. Herbut, V. Juricic, and O. Vafek, Phys. Rev. Lett. 100, 046403 (2008).

${ }^{13}$ M. Neek-Amal and F. M. Peeters, Phys. Rev. B 82, 085432 (2010). 
${ }^{14}$ L. Karssemeijer, Master's thesis, Institute for Molecules and Materials (Radboud University, Nijmegen, 2010).

${ }^{15}$ N. Mounet and N. Marzari, Phys. Rev. B 71, 205214 (2005).

${ }^{16}$ A. Fasolino, J. H. Los, and M. I. Katsnelson, Nat. Mater. 6, 858 (2007).

${ }^{17}$ W. Bao, F. Miao, Z. Chen, H. Zhang, W. Jang, C. Dames, and C. Ning Lau, Nature Nanotech. 4, 562 (2009).
${ }^{18}$ M. Neek-Amal and F. M. Peeters, J. Phys. Condens. Matter 23, 045002 (2011).

${ }^{19}$ M. Neek-Amal, R. Asgari, and M. R. Rahimi Tabar, Nanotechnology 20, 135602 (2009).

${ }^{20}$ A. Bhattacharya, S. Bhattacharya, C. Majumder, and G. P. Das, Phys. Rev. B 83, 033404 (2011).

${ }^{21}$ K. V. Zakharchenko, M. I. Katsnelson, and A. Fasolino, Phys. Rev. Lett. 102, 046808 (2009). 